\title{
A IMPORTÂNCIA RELATIVA DO ATLÂNTICO TROPICAL SUL E PACÍFICO LESTE NA VARIABILIDADE DE PRECIPITAÇÃO DO NORDESTE DO BRASIL
}

\author{
RITA VALÉRIA ANDREOLI ${ }^{1}$ e MARY TOSHIE KAYANO ${ }^{2}$ \\ ${ }^{1,2}$ Instituto Nacional de Pesquisas Espaciais, Centro de Previsão de Tempo e Estudos Climáticos. \\ Avenida dos Astronautas, 1758, CEP: 12227-010, São José dos Campos, SP, Brasil. \\ E-mail:1'rita@cptec.inpe.br, ${ }^{2}$ mary@cptec.inpe.br.
}

Recebido Maio 2005 - Aceito Novembro 2005

\begin{abstract}
RESUMO
Anomalias de precipitação no Nordeste do Brasil (NEB) são re-examinadas considerando-se os efeitos do El Niño-Oscilação Sul (ENOS) e do Atlântico Tropical (AT) na estação anterior à estação chuvosa do NEB. Na ausência de anomalias de Temperatura da Superfície do Mar (ATSMs) significativas no AT, durante a fase inicial e de desenvolvimento do ENOS, em DJF as anomalias na precipitação possivelmente são ocasionadas por padrões de teleconexões associados ao rearranjamento da célula de Walker, e em MAM, por padrões de teleconexões extratropicais. Simultâneos ENOS e ATSMs significativas no AT atuam para aumentar ou diminuir as anomalias de precipitação. ATSMs de mesmo sinal no ATS e Pacífico leste enfraquecem os padrões de precipitação no NEB. Quando as ATSMs do ATS são não significativas ou com magnitudes equivalentes às do ATN, porém com sinal oposto das ATSMs do Pacífico leste, as ATSMs do ATN e as anomalias da circulação atmosférica relacionada ao ENOS conduzem ao fortalecimento dos padrões anômalos de precipitação. Independente do ENOS, a influência do AT na variabilidade de precipitação é mais marcante para o ATS. Um aspecto relevante apresentado aqui é o papel do ATS na variabilidade de precipitação do NEB, cujo monitoramento é essencial para prospecções climáticas sazonais.
\end{abstract}

Palavras-chave: Nordeste do Brasil, precipitação, temperatura da superfície do mar, Pacífico tropical, Atlântico Tropical

\begin{abstract}
THE RELATIVE IMPORTANCE OF THE TROPICAL SOUTH ATLANTIC AND EASTERN PACIFIC ON RAINFALL VARIABILITY IN THE NORTHEAST BRAZIL.

Rainfall anomaly patterns over Northeastern Brazil (NEB) are re-examined considering the effects of the El Niño/Southern Oscillation (ENSO) and the Tropical Atlantic (TA) during the season preceding the northern NEB rainy season. In the absence of significant sea surface temperature anomalies (SSTAs) in TA, during the initial and development phases of the ENSO, precipitation anomalies in DJF are possibly due to teleconnection patterns associated with the rearrangement of the Walker cell, and in MAM, due to the extratropical teleconnection patterns. Simultaneous ENSO and significant SSTAs in the TA act to reinforce or to inhibit the precipitation anomalies. The same signal SSTAs in the tropical south Atlantic (TSA) and in the eastern Pacific weaken the precipitation pattern over NEB. When the SSTAs in the TSA are non-significant or with equivalent magnitudes of those in the ATN, but with opposite signal of the SSTAs in the eastern Pacific, the SSTAs in the ATN and the ENSO-related atmospheric circulation leads to reinforced anomalous precipitation patterns. Independent of the ENSO, the TA influence in the precipitation variability is remarkable for the TSA. The relevant aspect presented here is the important role of the TSA in the NEB precipitation variability. Thus, the TSA monitoring is essential for seasonal climate forecasting purposes.
\end{abstract}

Keywords: Northeastern Brazil, precipitation, sea surface temperature, tropical Pacific, tropical Atlantic. 


\section{INTRODUÇÃO}

Em um estudo pioneiro, Walker (1928) documentou uma notável coincidência do aquecimento anômalo das águas superficiais no Pacífico equatorial e as secas do nordeste do Brasil (NEB). Desde então, um grande número de estudos que buscam um melhor entendimento dos mecanismos que causam as anomalias climáticas sobre o NEB têm focalizado no papel que o fenômeno El Niño-Oscilação Sul (ENOS) exerce em tais anomalias. A componente oceânica do ENOS caracteriza-se pela ocorrência de Temperatura da Superfície do Mar (TSM) no Pacífico equatorial central e leste anomalamente positivas (El Niño) em uma fase e negativas (La Niña) na fase oposta, dinamicamente relacionada à Oscilação Sul, que se manifesta como uma gangorra barométrica com centros de ação na Indonésia e no Pacífico sudeste (Rasmusson e Carpenter, 1982.).

Vários estudos sugeriram que sob condições de El Niño, a circulação de Walker é deslocada para leste, tal que seu ramo ascendente localiza-se sobre o oceano Pacífico central e leste onde a convecção é intensificada, e seu ramo descendente localiza-se sobre o NEB e Atlântico Tropical (AT) adjacente, onde a convecção é inibida (Kousky et al., 1984; Kayano et al., 1988; Ropelewski e Halpert, 1987 e 1989). Assim, as secas severas (chuvas excessivas) no NEB, objeto de inúmeros estudos por seus impactos sociais e econômicos, têm sido relacionadas à ocorrência dos eventos El Niño (La Niña). Entretanto, Kane (1997) mostrou que dos 46 El Niño (fortes e moderados) do período de 1849-1992, somente 21 (45\%) estiveram associados a secas severas em Fortaleza.

Enquanto as ocorrências simultâneas de El Niño e secas no NEB restringem-se a determinados eventos, a precipitação nessa região tem sido, por outro lado, fortemente relacionada às anomalias de TSM (ATSMs) no AT. Moura e Shukla (1981) mostraram que para alguns anos, os eventos de secas do NEB estão associados a um dipolo meridional de ATSMs no AT. Eles sugeriram que as condições dinâmicas e termodinâmicas associadas ao dipolo meridional de ATSMs interferem sensivelmente na posição e intensidade da Zona de Convergência Intertropical (ZCIT) que, por sua vez, influencia a precipitação no NEB. Este modo envolve variações de TSM em ambos os hemisférios, em escalas de tempo sazonal, interanual e decadal (Servain, 1991). Chang et al. (1997) atribuíram essa variabilidade a interações oceano-atmosfera com um "feedback" positivo entre os fluxos de calor induzidos pelo vento e TSM.

No entanto, vários autores sugeriram que as componentes norte e sul do dipolo não estão dinamicamente acopladas, para as diversas escalas temporais, da sazonal à decadal (Houghton e Tourre, 1992; Enfield e Mayer, 1997; Enfield et al., 1999; Mo e Häkkinen, 2001; Andreoli e Kayano, 2004). De fato, alguns estudos têm mostrado ao invés de um dipolo, um gradiente meri- dional de ATSMs que cruza o equador relacionado a variações de precipitação (Hastenrath e Heller, 1977; Nobre e Shukla, 1996). Esse modo relaciona-se hidrostaticamente aos campos de pressão ao nível do mar sobre o Atlântico equatorial e afeta o deslocamento meridional da ZCIT.

Tanto o El Niño como o gradiente inter-hemisférico de ATSMs no AT são fatores importantes que modulam a precipitação do NEB. Isso tem motivado diversas pesquisas sobre as relações entre as TSM dos Oceanos Pacífico e Atlântico e a precipitação do NEB (Uvo et al., 1998; Saravanan e Chang, 2000; Pezzi e Cavalcanti, 2001; Giannini, et al., 2004; Andreoli et al., 2004; Kayano e Andreoli, 2004; Ambrizzi et al., 2004; Souza et al., 2004). Uma interpretação amplamente aceita é que a variabilidade de TSM do AT seja a forçante dominante das anomalias de precipitação no NEB, enquanto que a influência remota do Pacífico, em determinadas ocasiões pode reforçar estas anomalias, mas em outras ocasiões pode ter efeitos opostos, de modo que estas anomalias sejam enfraquecidas (Uvo et al., 1998; Pezzi e Cavalcanti, 2001; Ambrizzi et al., 2004; Souza et al., 2004). Um outro aspecto importante é que a previsibilidade da precipitação no NEB é dependente da distribuição de ATSMs no Pacífico tropical e AT. Hastenrath (1990) e Hastenrath e Greishar (1993) mostraram que a variabilidade de precipitação sobre o NEB é previsível com alguns meses de antecedência, usando uma combinação de ATSMs do Pacífico e do AT.

Em adição, Kane (1992), usando índices de TSM definidos por Servain (1991), mostrou evidências de que as variações de precipitação em Fortaleza são melhor relacionadas com as ATSMs do ATS do que com as do ATN. A independência do papel do ATS na precipitação do NEB, também notada por Kayano e Andreoli (2004) para a escala decadal, é provavelmente uma indicação da variabilidade de TSM independente em ambos os hemisférios do AT como sugerida anteriormente. Enfield et al. (1999) encontraram indicações de que um modo com ATSMs anti-simétricas relativa à ZCIT, tem probabilidade de ocorrência de 12-15\%. Desta forma, a caracterização da relação entre ATSMs do AT e a variabilidade de precipitação com um índice, como o definido por Servain (1991), pode ser incompleta desde que as flutuações em cada setor (norte e sul) do AT não são temporalmente coerentes.

Dada a importância da variabilidade de TSM na previsibilidade de precipitação do NEB, as relações entre as ATSMs dos oceanos Pacífico e AT e os campos anômalos de precipitação no NEB são re-examinadas considerando-se os efeitos combinados e isolados das ATSMs nestas bacias oceânicas na estação que antecede a estação de chuvosa no norte do NEB. Gianini et al. (2004) mostraram evidências que a relação entre o ENOS e a variabilidade do AT durante a estação chuvosa do NEB pode ser afetada pela pré-condição de até seis meses de 
antecedência das ATSMs no AT. Além disso, seus resultados indicaram que a pré-condição no ATS pode ser tão importante quanto a do ATN na determinação do gradiente meridional de TSM, e conseqüentemente da anomalia de precipitação no NEB, em MAM.

\section{DADOS E METODOLOGIA}

Os dados usados consistem de campos globais mensais de TSM, e precipitação sobre a América do Sul. Os dados de TSM consistem de séries temporais reconstruídas por Smith e Reynolds (2003) em cada ponto de grade, com uma resolução espacial de $2^{\circ}$ de latitude por $2^{\circ}$ em longitude para o período de 1854 a 2000. Séries de anomalias mensais de precipitação sobre a América do Sul limitadas entre o equador, $20^{\circ} \mathrm{S}, 50^{\circ} \mathrm{W}$ and $30^{\circ} \mathrm{W}$, para o período de 1912-1999 são extraídas de várias fontes levando em consideração a quantidade de informações disponíveis para esse período. As fontes dos dados de precipitação são: a Superintendência do Desenvolvimento do Nordeste (SUDENE), o Instituto Nacional de Meteorologia (INMET) e a Agência Nacional de Energia Elétrica (ANEEL). Séries de precipitação sobre esta região, selecionadas a partir de um conjunto de dados históricos, 'gu23wld0098.dat' (version 1.0), construídos em pontos de grade, com uma resolução espacial de $2,5^{\circ} \mathrm{em}$ latitude por $3,75^{\circ} \mathrm{em}$ longitude, para o período de 1900 a 1998, e disponibilizadas pelo Dr. Mike Hulme são também usadas. Estes dados foram gerados a partir de séries históricas de precipitação medidas em estações de superfície. A descrição desses dados pode ser encontrada em Hulme $(1992,1994)$ e em Hulme et al. (1998). A Figura 1 ilustra as localizações dos pontos de grade e das estações utilizadas.

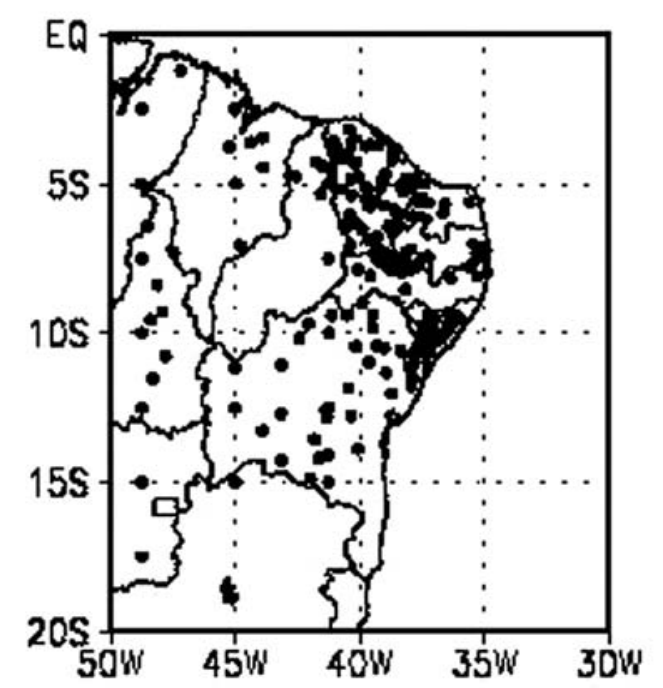

Figura 1 - Localizações das estações e pontos de grade das séries de precipitação utilizadas.
Anomalias mensais são calculadas removendo-se o ciclo anual usando a climatologia mensal do período de 1912 a 1999. As anomalias de precipitação são normalizadas pelos desvios padrão das séries de anomalias. Os eventos ENOS são selecionados através do índice do Niño-3, definido como a média espacial das ATSMs sobre a região delimitada em $6^{\circ} \mathrm{N}, 6^{\circ} \mathrm{S}, 150^{\circ} \mathrm{W}$ e $90^{\circ} \mathrm{W}$. Valores do Niño-3 médios para DJF maiores que $0.9^{\circ} \mathrm{C}$ (menores que $-0.7^{\circ} \mathrm{C}$ ), identificam o ano que corresponde a dezembro como sendo o inicial $(\operatorname{ano}(0))$ do evento quente ou frio do ENOS. O ano seguinte $(\mathrm{ano}(+))$ se refere à fase madura do evento. $\mathrm{O}$ limiar para os eventos frios é menor para refletir a assimetria na distribuição do Niño-3. Índices mensais que representam as variações de TSM nos setores norte e sul do AT são obtidos calculando-se a média das ATSMs na região delimitada em $0^{\circ}, 20^{\circ} \mathrm{N}, 60^{\circ} \mathrm{W}, 20^{\circ} \mathrm{W}$, para o setor norte e em $0^{\circ}, 20^{\circ} \mathrm{S}, 30^{\circ} \mathrm{W}, 10^{\circ} \mathrm{E}$, para o setor sul.

A diferença entre os índices do ATN e ATS descreve o gradiente inter-hemisférico de TSM no AT (GRAD). Como o interesse é estudar os efeitos das ATSMs dos oceanos Pacífico e AT ocorrendo na estação que antecede a estação chuvosa do NEB os eventos de GRAD positivo e negativo no AT são selecionados considerando a média sazonal do índice GRAD para DJF. O valor médio sazonal maior (menor) que + (-) 0.8 desvios padrão $\left(\sigma=0.25^{\circ} \mathrm{C}\right)$ identifica os GRAD positivos (negativos) no AT. A Tabela 1 lista os anos com eventos quentes/frios no Pacífico e os GRAD positivos/negativos (GRADP, GRADN) no AT ocorrendo simultaneamente ou isoladamente para o período de 1912 a 1999. Em seguida, é feita uma classificação refinada dos eventos GRAD considerando as amplitudes das ATSMs dos ATN e ATS. A Tabela 2 lista os anos com eventos GRAD definidos principalmente por ATSMs com valores superiores a um desvio padrão no ATN e abaixo desse limiar no ATS (GRAD, ATN), valores superiores a um desvio padrão no ATS e inferiores no ATN (GRAD, ATS) e quando as ATSMs do ATN e ATS apresentam magnitudes equivalentes, porém com sinal contrário (GRAD, ATN_ATS). Análises para eventos quentes/frios no Pacífico separadamente para a classificação mais refinada dos eventos GRAD não são feitas devido ao pequeno número de eventos. No entanto, vale salientar que nos casos de El Niño e GRAD negativo (El Niño/c_GRADN) e de La Niña e GRAD positivo (La Niña/c_GRADP), as ATSMs que definem o GRAD são devido principalmente às ATSMs com valores superiores a um desvio padrão na região do ATS e inferiores a esse valor no ATN. Nos casos de La Niña e GRAD negativo (La Niña/c_GRADN), o GRADN é definido por ATSMs do ATN e do ATS com valores aproximados porém de sinais opostos. Nos casos de El Niño e GRAD positivo (El Niño/c_GRADP), um evento é definido por ATSMs do ATN maiores que um desvio padrão e abaixo desse valor no ATS, outro por ATSMs do ATS maiores que um desvio padrão e inferiores a esse valor no ATN e o outro por ATSMs do ATN e ATS de magnitudes equivalentes e sinais opostos. 
A análise de compósitos é usada para obter os campos espaciais de anomalias de precipitação no NEB e TSM durante os períodos de DJF e MAM dos casos listados nas Tabelas 1 e 2 . Para obter a significância estatística desses campos, aplica-se o teste-t de Student, considerando o número de graus de liberdade como número de eventos selecionados.

\section{RESULTADOS}

Como esperado, os compósitos médios mensais de ATSMs, normalizados pelo desvio padrão, para as regiões do Pacífico e Atlântico entre $60^{\circ} \mathrm{N}-60^{\circ} \mathrm{S}$, para os períodos de DJF e MAM (Figura 2) mostram padrões de ATSMs aproximadamente opostos entre as fases quente e fria do ENOS. As composições para o El Niño/s_GRAD (La Niña/s_GRAD) apresentam ATSMs positivas (negativas) ao longo da costa do continente Americano e no Pacífico central e leste entre $15^{\circ} \mathrm{N}$ e $15^{\circ} \mathrm{S}$, circundadas ao norte e sul por anomalias de sinal oposto. Os padrões de ATSMs para DJF assemelham aos padrões obtidos anteriormente durante o estágio maduro dos eventos de ENOS canônicos (Rasmusson e Carpenter, 1982). Para ambas as estações, as composições para casos de El Niño mostram pequenas ATSMs na região do AT, e as composições de La Niña apresentam anomalias significativas em uma região extensa do ATN. Esse padrão de ATSMs no AT representa um gradiente de TSM menos intenso do que aqueles selecionados anteriormente (Tabela 1), e tem sido relacionado aos eventos de La Niña em trabalhos anteriores (Enfield e Mayer, 1997).

Tabela 1 - Anos de eventos extremos no Pacífico e AT ocorrendo simultaneamente/ isoladamente para o período de 1912-1999.

\begin{tabular}{|c|c|c|c|c|c|c|c|}
\hline \multicolumn{2}{|c|}{ ENOS } & \multicolumn{2}{|c|}{ EI Niño/GRAD } & \multicolumn{2}{|c|}{ La Niña/GRAD } & \multicolumn{2}{|c|}{ GRAD } \\
\hline $\begin{array}{l}\text { El Niño/ } \\
\text { s_GRAD }\end{array}$ & $\begin{array}{l}\text { La Niña/ } \\
\text { s_GRAD }\end{array}$ & $\begin{array}{c}\text { EI Niño/ } \\
\text { c_GRADP }\end{array}$ & $\begin{array}{c}\text { EI Niño/ } \\
\text { c_GRADN }\end{array}$ & $\begin{array}{c}\text { La Niña/ } \\
\text { c_GRADP }\end{array}$ & $\begin{array}{c}\text { La Niña/ } \\
\text { c_GRADN }\end{array}$ & GRADP & GRADN \\
\hline 1913 & 1916 & 1957 & 1940 & 1942 & 1973 & 1917 & 1912 \\
\hline 1914 & 1922 & 1965 & 1951 & 1954 & 1984 & 1931 & 1915 \\
\hline 1918 & 1924 & 1991 & 1972 & 1955 & 1988 & 1935 & 1919 \\
\hline 1925 & 1933 & & 1982 & 1996 & & 1937 & 1920 \\
\hline 1930 & 1949 & & 1997 & & & 1950 & 1923 \\
\hline 1939 & 1967 & & & & & 1952 & 1934 \\
\hline 1941 & 1970 & & & & & 1953 & 1983 \\
\hline 1986 & 1975 & & & & & 1959 & 1985 \\
\hline & 1998 & & & & & 1962 & 1987 \\
\hline & & & & & & 1969 & 1993 \\
\hline & & & & & & 1977 & 1994 \\
\hline & & & & & & 1979 & \\
\hline & & & & & & 1980 & \\
\hline & & & & & & 1981 & \\
\hline
\end{tabular}

Tabela 2 - Classificação refinada dos eventos GRAD com respeito às amplitudes das ATSMs do ATN e ATS.

\begin{tabular}{cccccc}
\hline \multicolumn{2}{c}{ GRAD Negativo } & \multicolumn{3}{c}{ GRAD Positivo } \\
\cline { 5 - 6 } GRADN/ATN & GRADN/ATS & GRADP/ATN & GRADP/ATS & GRADP/ATN_ATS \\
\hline 1912 & 1915 & 1935 & 1917 & 1931 \\
1919 & 1923 & 1937 & 1950 & 1952 \\
1985 & 1934 & 1962 & 1953 & 1969 \\
1993 & 1983 & & 1959 & 1981 \\
& 1987 & & 1977 & \\
& 1994 & & 1979 & \\
\hline
\end{tabular}


Os campos correspondentes de anomalias de precipitação são apresentados na Figura 3. Estes campos mostram configurações de sinais aproximadamente opostos entre as duas fases do ENOS (Figura 3a e 3b, 3c e 3d). Composições de El Niño/s_GRAD (La Niña/s_GRAD) mostram anomalias negativas (positivas) no norte do NEB e anomalias de sinal oposto ao sul. Em DJF, o efeito do El Niño/s_GRAD na precipitação é mais significativo na parte sudoeste da região de estudo (Goiás e centro oeste de Minas Gerais), e o da La Niña/s_GRAD é uma estrutura dipolar com anomalias não significativas positivas ao norte (Alagoas, Pernambuco, Paraíba, Rio Grande do Norte, Ceará e extremo norte do Piauí e Maranhão) e negativas ao sul (extremo sul do Piauí e Maranhão, Bahia, leste do Tocantins e Goiás e norte de Minas Gerais). Nesta estação as anomalias de precipitação podem ser atribuídas primeiramente a ação da circulação de Walker anômala ajustada através do rearranjamento da convecção no Pacífico equatorial leste como discutido em trabalhos anteriores (Kousky et al., 1984; Kayano et al., 1988; Ropelewski e Halpert, 1987 e 1989). Isto porque a influência do ENOS no padrão de TSM do ATN somente tem início depois da fase madura do ENOS, ou seja em MAM (Enfield e Mayer, 1997). Em MAM, as anomalias positivas (negativas) no sul de $10^{\circ} \mathrm{S}$ são enfraquecidas. No entanto, anomalias significativas estendem-se de sudeste para noroeste ao sul de $10^{\circ} \mathrm{S}$ para composições de La Niña/s_GRAD. Na região norte da área de estudo, que inclui os Estados de Alagoas, Pernambuco, Paraíba, Rio Grande do Norte, Ceará, Piauí e Maranhão as anomalias negativas (positivas) se intensificam para as composições de El Niño/s GRAD e La Niña/s GRAD. Essas anomalias podem estar associadas à influência do ENOS na definição das ATSMs do ATN através de um "caminho atmosférico" semelhante ao padrão de teleconexão Pacífico-América do Norte (PNA) (Horel e Wallace, 1981) gerando um aquecimento no ATN, que conseqüentemente cria um gradiente meridional de TSM que afeta a posição da ZCIT como discutido por Nobre e Shukla (1996).

O padrão anômalo de ATSMs para os eventos El Niño e GRAD positivo ou negativo (El Niño/c_GRADP, El Niño/c GRADN) no AT são mostrados na Figura 4. Em DJF, para os eventos de El Niño/c GRADP na região do Pacífico, ATSMs positivas significativas restritas à região equatorial central e leste são circundadas por anomalias de sinal oposto ao norte e sul. No AT, ATSMs significativas positivas (negativas) encontram-se no ATN próximo à costa da África (Atlântico equatorial). Em MAM, a região de ATSMs significativas positivas no Pacífico restringe-se à parte equatorial central, e no AT as
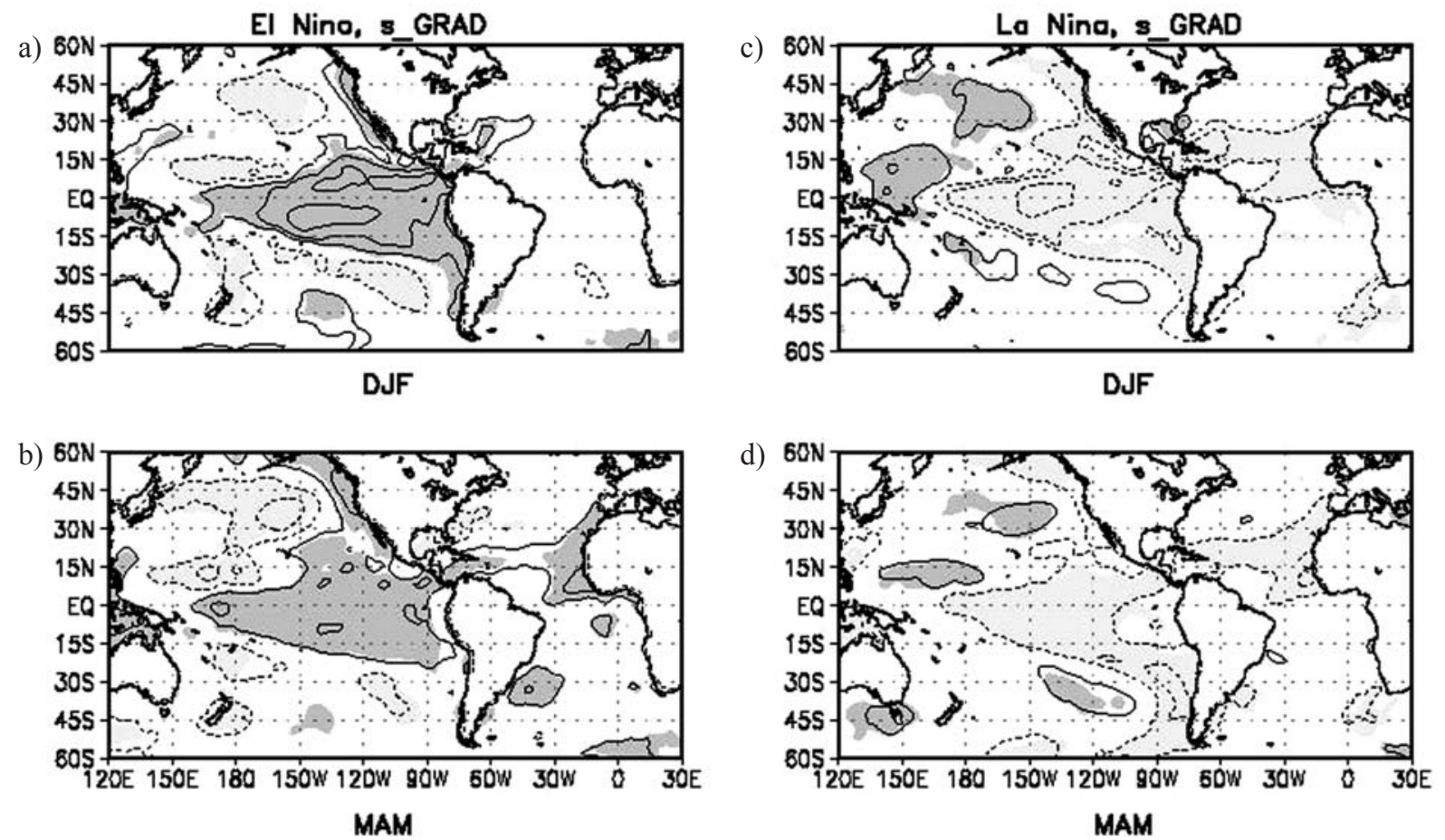

Figura 2 - Composições médias mensais de ATSMs normalizadas pelo desvio padrão para os eventos de El Niño/s_GRAD em (a) DJF e (b) MAM; eventos de La Niña/s GRAD em (c) DJF e (d) MAM. O contorno zero é omitido. Intervalo de contorno é 0,5 desvio padrão com contornos positivos (negativos) contínuos (tracejados). Sombreados indicam valores significativos no nível de confiança de $95 \%$. 

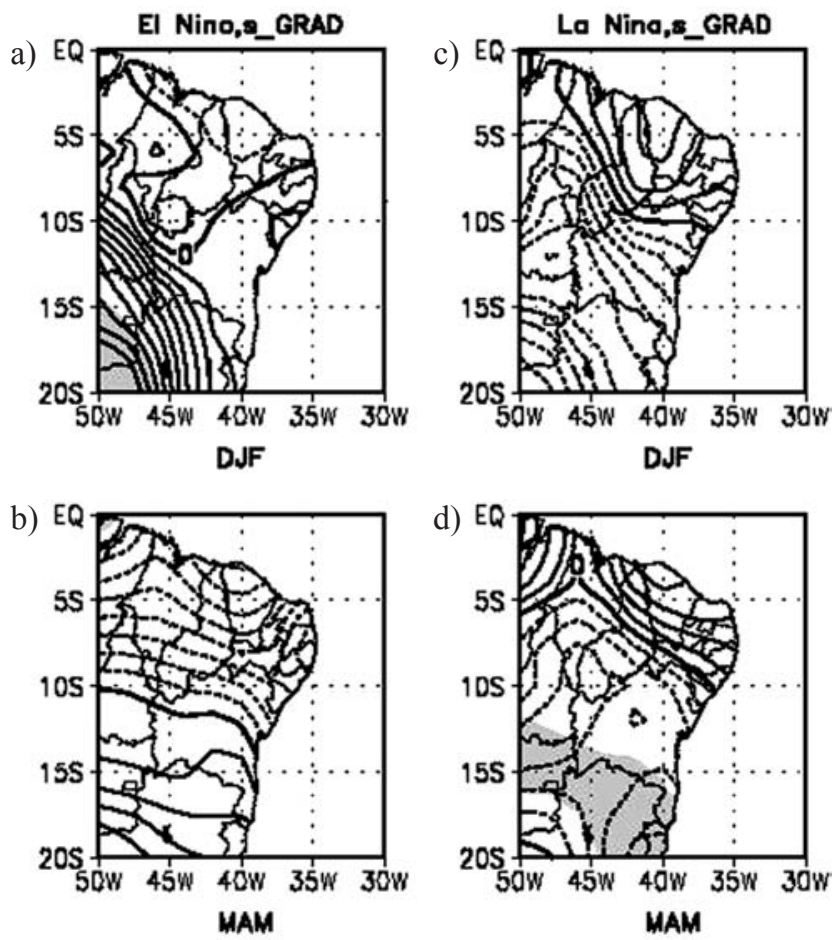

Figura 3 - Médias de anomalias mensais de precipitação normalizadas para composições de: El Niño/s_GRAD em (a) DJF e em (b) MAM; e de La Niña/s_GRAD em (c) DJF e em (d) MAM. Intervalo de contorno é de $0, \overline{1}$ desvio padrão, com contornos positivos (negativos) contínuos (tracejados). Sombreados indicam valores significativos no nível de confiança de $95 \%$.
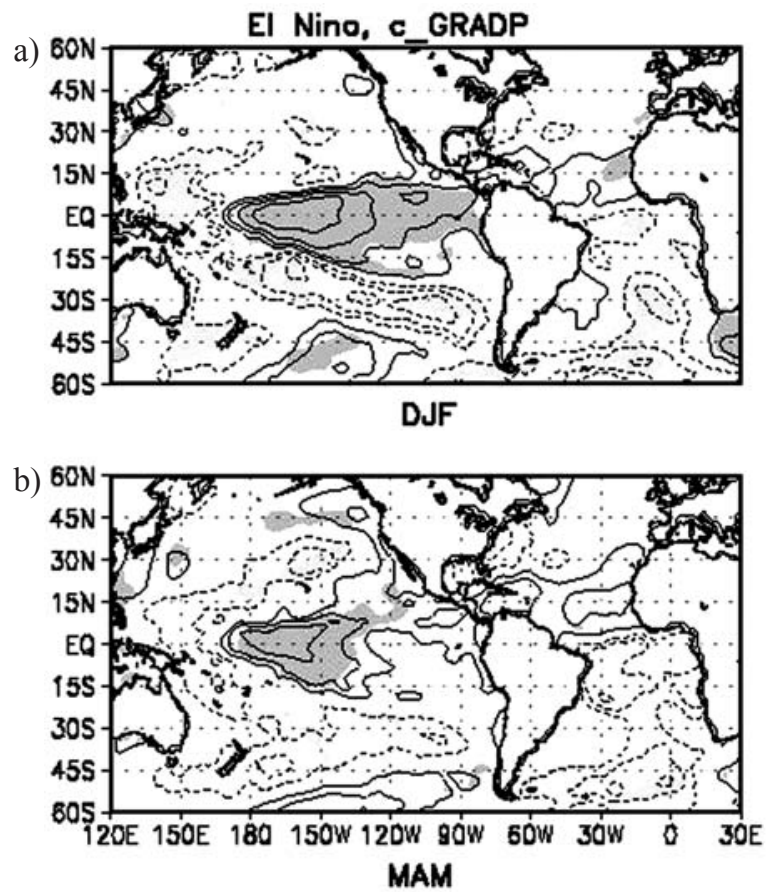

anomalias intensificam-se mantendo o gradiente meridional de TSM nessa região. Por outro lado, o padrão de TSM para o caso de El Niño/c_GRADN não apresenta uma estrutura com ATSMs de sinais opostos ao norte e sul do equador no AT. Em DJF, anomalias negativas significativas cobrem a região do Pacífico equatorial central e leste e grande parte do Atlântico equatorial e ATS. Em MAM, o padrão de anomalias é enfraquecido.

Os campos de anomalias de precipitação associados aos eventos El Niño/c_GRADP e El Niño/c_GRADN são mostrados na Figura 5. Os sinais mais significativos aparecem para a composição com ocorrências simultâneas de El Niño/c_GRADP (Figuras 5a e 5b). Nesse caso, há uma intensificação das anomalias negativas no norte do NEB em MAM em relação ao período de DJF e valores significativos restringem-se ao Estado do Rio Grande do Norte e uma pequena região na parte norte do Maranhão (Figura 5b). Isto pode ser explicado pelo enfraquecimento das ATSMs na região do Pacífico equatorial leste enquanto as ATSMs no AT tem uma ligeira intensificação de DJF para MAM. A permanência do gradiente meridional de ATSMs no AT favorece o aumento de precipitação nessa região (Nobre e Shukla, 1996). Para as composições de El Niño/c_GRADN, as anomalias no norte do NEB são menos intensas em relação aos eventos El Niño/c_GRADP e não significativas em DJF e MAM (Figuras 5c e 5d). Analisando as composições de ATSMs associadas a esses eventos (Figura 4c e 4d) notam-se em DJF, ATSMs no ATS de sinais opostos àquelas do Pacífico,
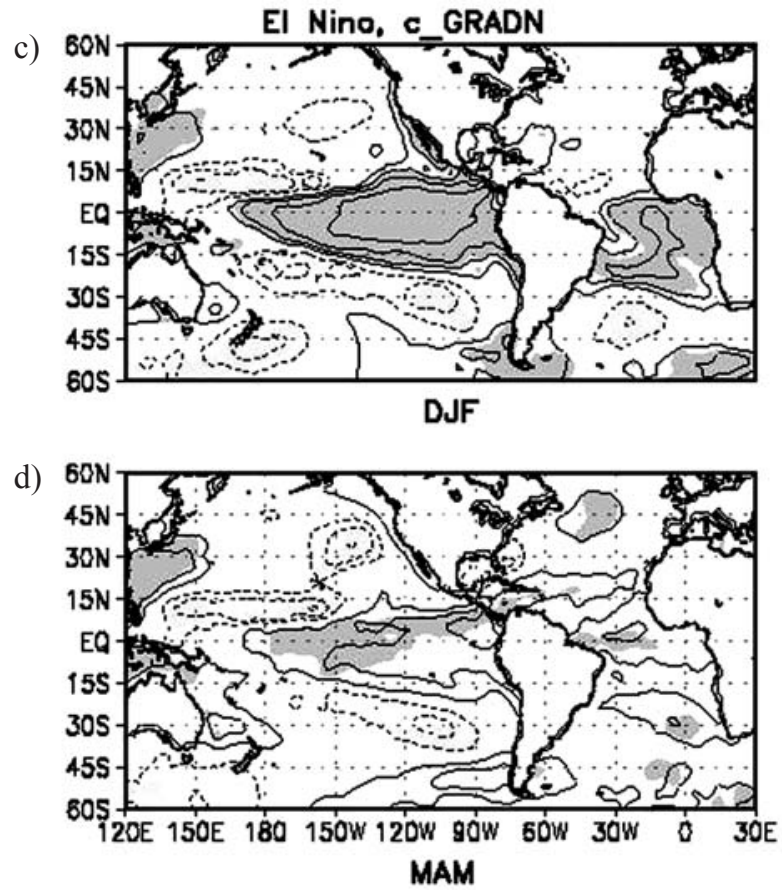

Figura 4 - Composições médias mensais de ATSMs normalizadas pelo desvio padrão para os eventos de El Niño/c_GRAP em (a) DJF e (b) MAM e El Niño/c_GRAN em (c) DJF e (d) MAM. A convenção gráfica é igual a da Figura 2. 
as quais têm efeitos opostos, o que pode explicar o enfraquecimento das anomalias negativas de precipitação. No caso do El Niño/c GRADN, há um enfraquecimento das ATSMs em toda a área de estudo em MAM e conseqüentemente, um enfraquecimento do gradiente meridional de TSM no AT.

As composições de ATSMs considerando os eventos de La Niña e do GRAD no AT (La Niña/c_GRADP e La Niña/c_GRADN) são apresentadas na Figura 6. As composições para os eventos de La Niña/c_GRADP caracterizam-se por ATSMs negativas na região do Pacífico equatorial e leste, no Atlântico equatorial e ATS, e por ATSMs positivas no ATN durante DJF (Figura 6a). Em MAM, há enfraquecimentos das ATSMs nas regiões do Pacífico e ATN, no entanto, o GRADP no AT contrasta com o esperado padrão de ATSMs associados aos eventos de La Niña (Enfield e Mayer, 1997). Para o caso de La Niña/c_GRADN é notada uma intensificação das ATSMs na região do ATN, como descrito anteriormente (Enfield e Mayer, 1997).

Os efeitos combinados da La Niña/c_GRAD na precipitação da região analisada mostram configurações inversas, para os casos de La Niña/c_GRADP (Figuras 7a e 7b) e La Niña/c_GRADN (Figuras 7c e 7d). Nesse último caso (Figura 7d), as anomalias positivas em MAM são consideravelmente maiores do que as correspondentes para a composição considerando os eventos de La Niña/s_GRAD mostrada na Figura 3d. As anomalias positivas significativas cobrem os
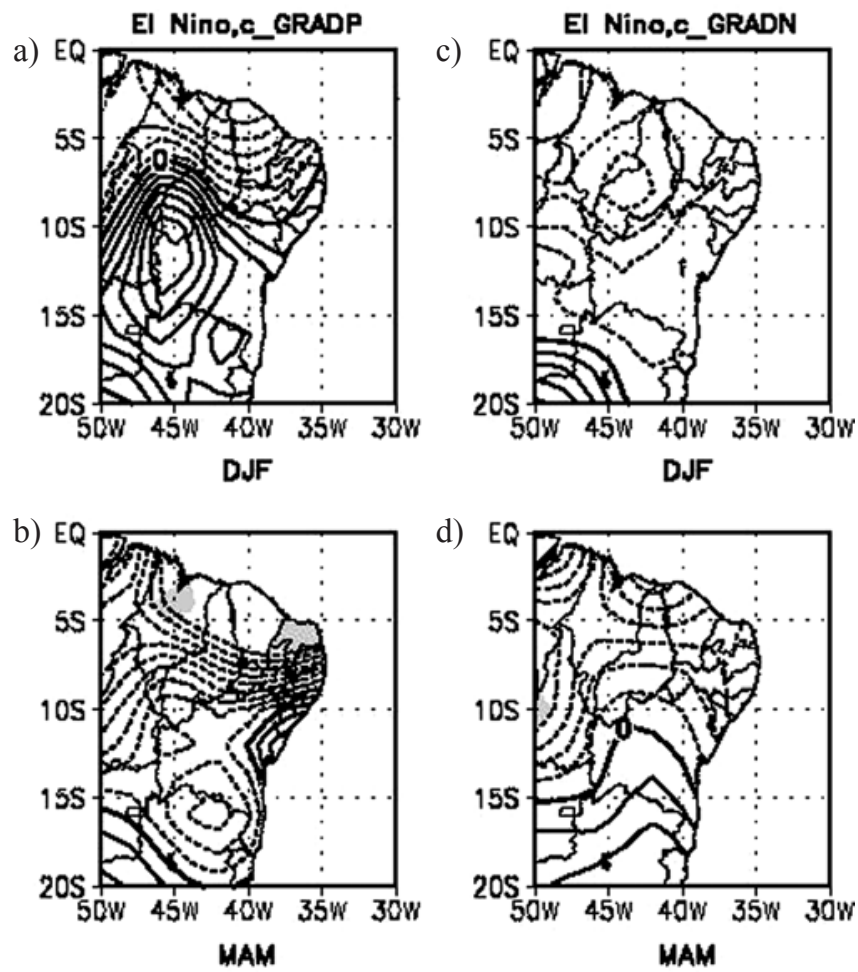

Figura 5 - Médias de anomalias mensais de precipitação para composições de: El Niño/c_GRADP em (a) DJF e em (b) MAM; e de El Niño/c_GRADN em (c) DJF em (d) MAM. A convenção gráfica é igual a da Figura 3.
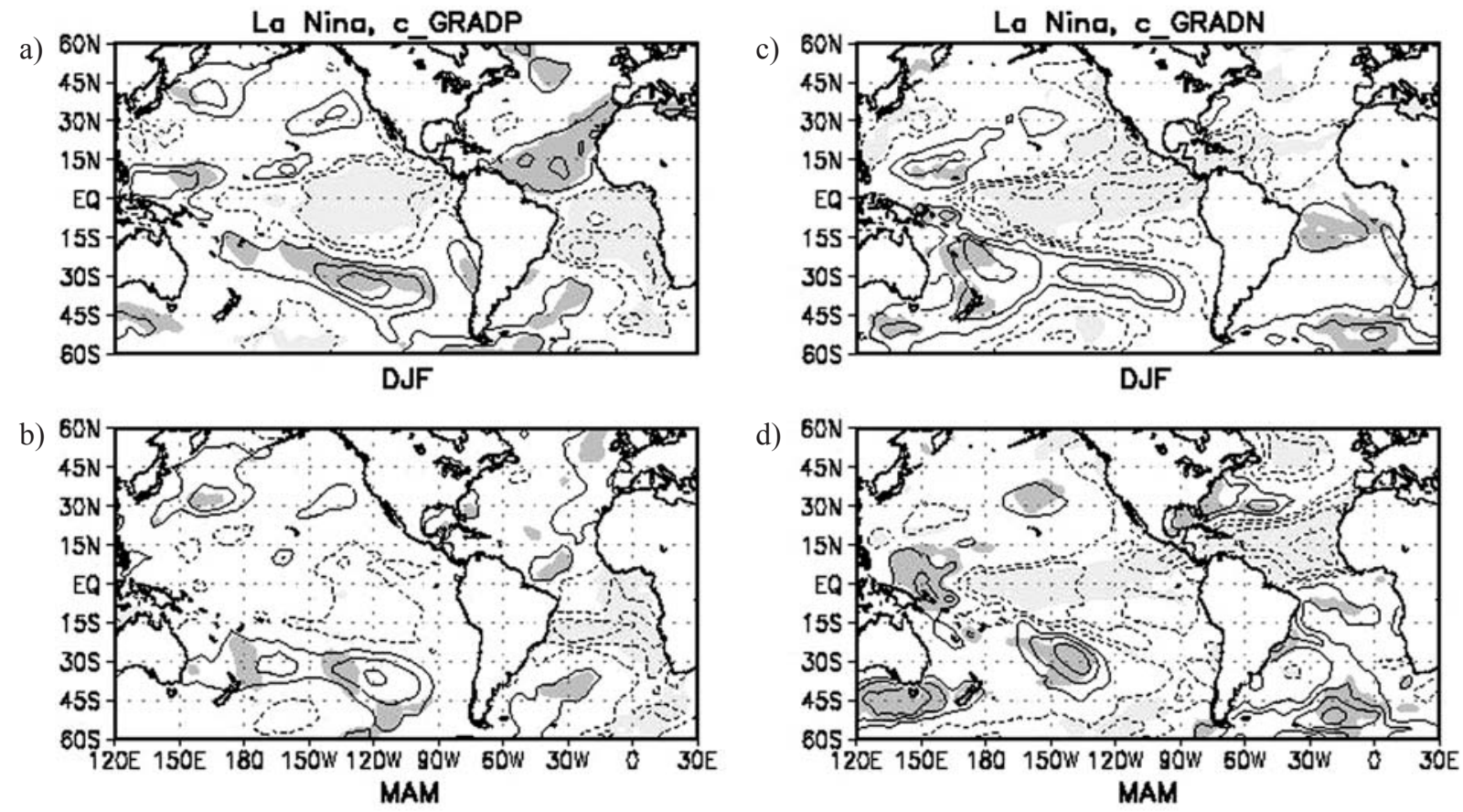

Figura 6 - Composições médias mensais de ATSMs normalizadas pelo desvio padrão para os eventos de La Niña/c_GRAP em (a) DJF e (b) MAM e La Niña/c_GRAN em (c) DJF e (d) MAM. A convenção gráfica é igual a da Figura 2. 
Estados de Alagoas, Pernambuco, Paraíba, Rio Grande do Norte, Ceará, regiões norte do Piauí e Maranhão. Neste caso, as ATSMs negativas no ATN e as anomalias na circulação atmosférica associadas aos eventos La Niña têm efeitos na precipitação no NEB que se somam, favorecendo um aumento anômalo da precipitação nesta região. Por outro lado, para o caso de La Niña/c_GRADP, anomalias negativas de precipitação não significativas são observadas cobrindo toda a região em DJF e em MAM anomalias negativas significativas são notadas no Estado do Maranhão (Figuras 7a e 7b). Isto é justificado pelo enfraquecimento das ATSMs no Pacífico equatorial leste de DJF para MAM (Figuras 6a e 6b) enquanto que as ATSMs no ATS permanecem fortes. Em conseqüência, as ATSMs no ATS agem contrariamente à influência dos padrões de teleconexões associados aos eventos de La Niña no AT e NEB, inibindo a convecção e então causando secas nessa região.

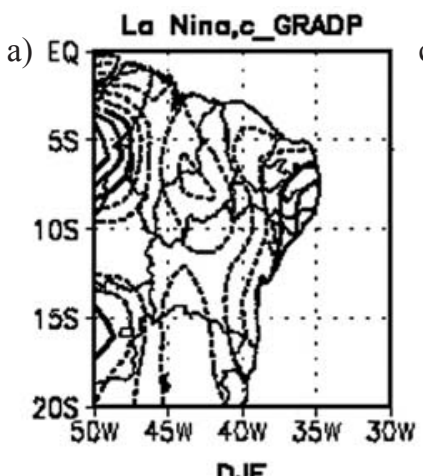

DJF

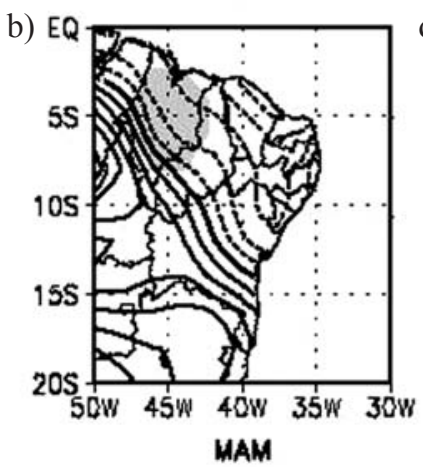

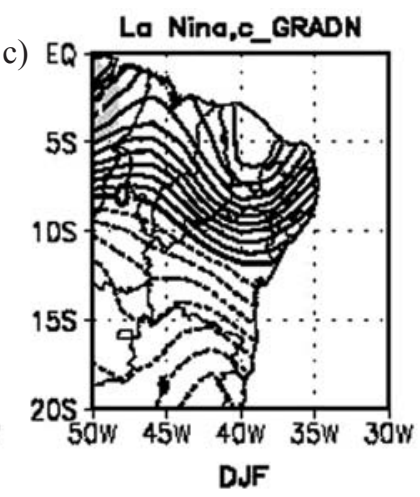

d) $E$

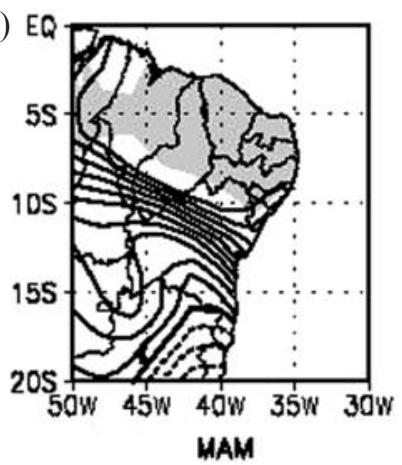

Figura 7 - Médias de anomalias mensais de precipitação para composições de: La Niña/c_GRADP em (a) DJF e (b) MAM; e de La Niña/c_GRADN em (c) DJF e (d) MAM. A convenção gráfica é igual a da Figura 3.

A Figura 8 mostra campos de ATSMs considerando somente os efeitos do GRAD no AT (GRAD/s_ENOS). Em DJF, anomalias positivas (negativas) significativas são encontradas cobrindo toda a região do ATN (ATS) e fracas ATSMs de sinal oposto no ATS (ATN) para o caso de GRADP/s_ENOS (GRADN/s_ENOS). Em MAM, as ATSMs na região do ATN restringem-se na parte leste do ATN enquanto no ATS há uma ligeira expansão das anomalias em toda a região do ATS para o caso de GRADP/s_ENOS. Para o caso do GRADN/s_ENOS, o padrão de DJF persiste em MAM.

Os campos de anomalias de precipitação associados a esses padrões de ATSMs são mostrados na Figura 9. Em DJF, para o GRADP/s_ENOS, anomalias negativas não significativas estendem-se por toda a parte norte, incluindo os Estados da Paraíba, Rio Grande do Norte, Ceará, norte do Piauí e sudoeste da região de estudo. Anomalias positivas não significativas restringem-se às partes central e sudeste da região de estudo (Figura 9a). As anomalias negativas intensificam-se em MAM com valores significativos nos Estados do Rio Grande do Norte, Ceará, e norte dos Estados do Piauí e Maranhão e leste do Pará (Figura 9b). No caso de GRADN/s_ENOS, as anomalias são muito fracas por toda a região em DJF, e em MAM anomalias positivas de precipitação cobrem toda a região com valores significativos ao norte de $7^{\circ} \mathrm{S}$ (Figuras 9c e 9d). Consistente, com resultados anteriores as anomalias positivas (negativas) de precipitação estão associados ao gradiente meridional de ATSMs no AT como discutido por Nobre e Shukla (1996).

Uma classificação dos eventos de GRAD positivo/negativo considerando amplitude das ATSMs nos setores norte e sul do AT é apresentada na Figura 10. Esta figura mostra campos de anomalias de precipitação relacionados aos GRADP/ATN (a), GRADN/ATN (b) e GRADP/ATS (c) e GRADN/ATS (d) para MAM. Observa-se que anomalias significativas (Figuras 10d) surgem somente para composições cujo GRAD é definido quando as ATSMs do ATS são maiores que um desvio padrão e ATSMs no ATN são inferiores a esse valor (Figura não mostrada). Neste caso as anomalias de precipitação atingem máximos valores de 1,4 desvios padrão. Esse resultado é consistente com o mapa de correlações de Moura e Shukla (1981), que encontraram correlações superiores a 0.7 entre a série de precipitação média de Fortaleza e Quixeramobim e as ATSMs do ATS que contrastam com máximas correlações negativas (-0.4) entre esta série e as ATSMs no ATN. A relação entre as ATSMs positivas do ATS e anomalias positivas de precipitação podem ser explicadas como segue: ATSMs positivas e conseqüentemente o aumento de evaporação sobre o oceano aumenta o fluxo de umidade convergente e precipitação sobre o NEB por que os alísios de sudeste fluem diretamente em direção ao NEB. Por outro lado, ATSMs negativas diminuem a evaporação e reduz a convecção como indicado por Markham e McLain (1977). Embora a relação entre as ATSMs do ATN e precipitação do NEB não apresenta valores significativos, Moura e Shukla (1981) argumentam que as ATSMs nessa região têm um efeito dinâmico muito importante para a precipitação do NEB. Enquanto as ATSMs positivas no ATN produzem intensa ZCIT e movimentos descendentes associados sobre o NEB, as ATSMs negativas neste setor oceânico não necessariamente induzem movimentos ascendentes e aumento de precipitação no NEB. 

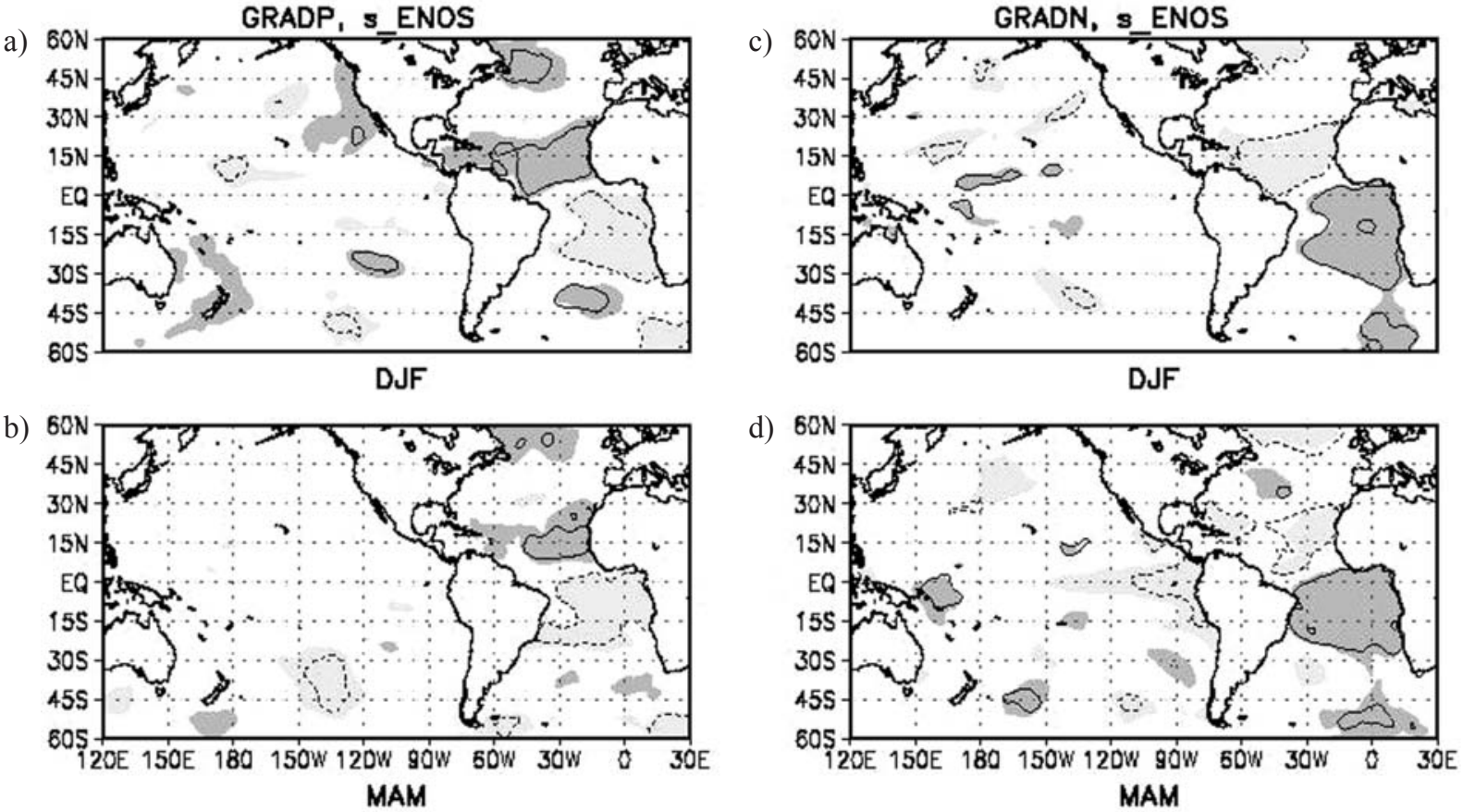

Figura 8 - Composições médias mensais de ATSMs normalizadas pelo desvio padrão para os eventos de GRADP/s_ENOS em (a) DJF e (b) MAM e GRADN/s_ENOS em (c) DJF e (d) MAM. A convenção gráfica é igual a da Figura 2.
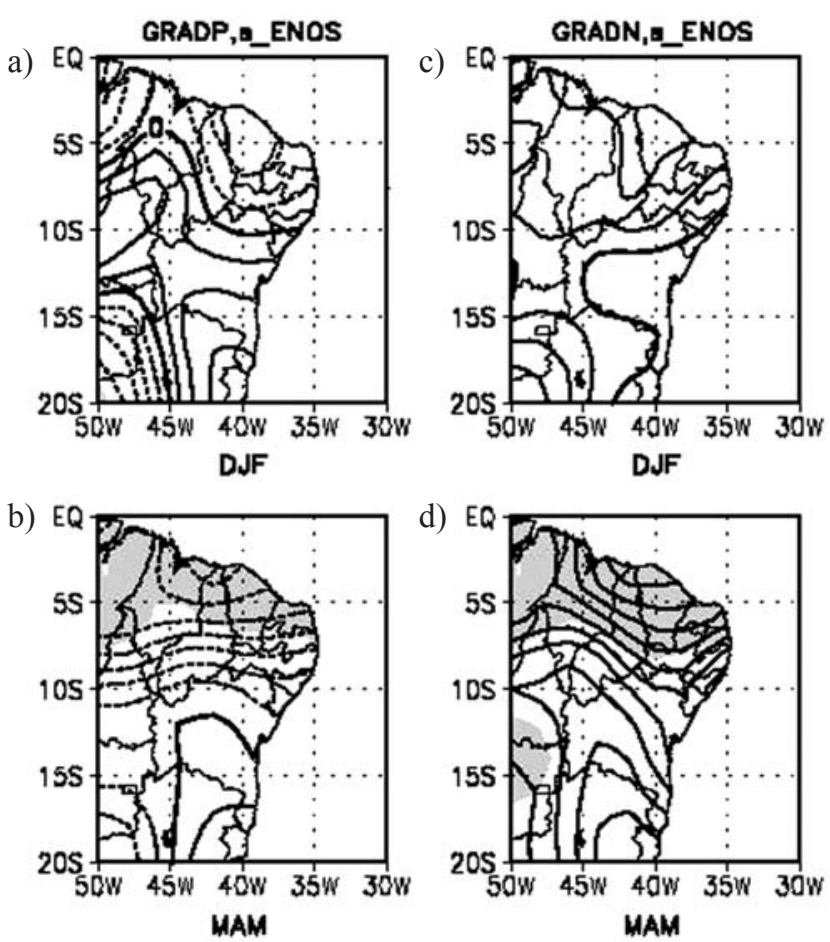

Figura 9 - Médias de anomalias mensais de precipitação para composições de: GRADP/s ENOS em (a) DJF, e (b) MAM; de GRADN/s_ENOS em (c) DJF e (d) MAM. A convenção gráfica é igual a da Figura 3.
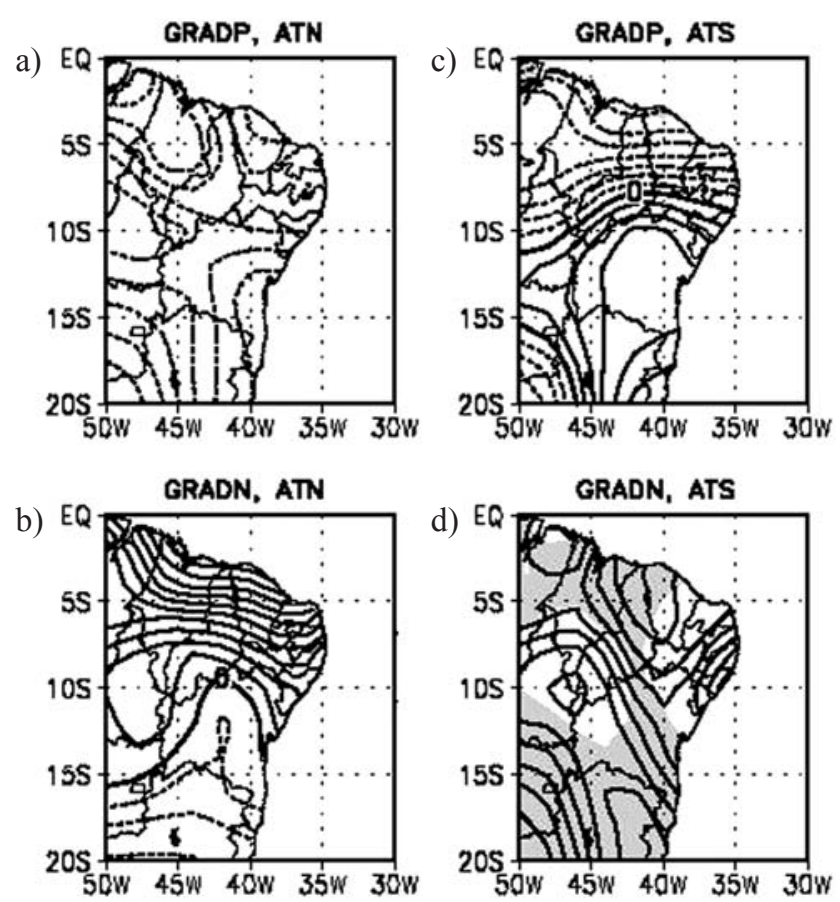

Figura 10 - Médias de anomalias mensais de precipitação em MAM para composições de (a) GRADP/ATN, (b) GRADN/ATN, (c) GRADP/ATS e (d) GRADN/ATS. A convenção gráfica é igual a da Figura 3. 
A Figura 11 mostra as composições de precipitação para GRAD definido quando as ATSMs do ATN e ATS apresentam magnitudes equivalentes, porém de sinais contrários. Somente a composição relacionada ao GRADP para MAM é apresentada. Composição para GRADN não é feita porque o número de eventos não é suficiente para calcular a significância estatística. É notado na Figura 11 um extensa área com anomalias negativas significativas de precipitação, cujas magnitudes são maiores do que nos mapas de anomalias de precipitação correspondentes aos eventos GRADP/ATN e a GRADN/ATN (Figuras 10a e 10c). O possível mecanismo associado a essa escassez de chuva no NEB é a intensificação da ZCIT ao norte do Equador, a qual ocorre em associação com ATSMs positivas, e o estabelecimento de movimento descendente sobre o NEB e áreas oceânicas adjacentes com ATSMs negativas, as quais reduzem a convecção e precipitação, como proposto por Moura e Shukla (1981). Segundo os autores, a ocorrência simultânea de ATSMs positivas no ATN e negativas no ATS reforçam esse mecanismo e conseqüentemente, as secas sobre o NEB. No entanto como mostrado em trabalhos anteriores (por ex., Enfield et al., 1999) a probabilidade de ocorrência desses eventos de dipolo é de $12 \%$, aproximadamente.

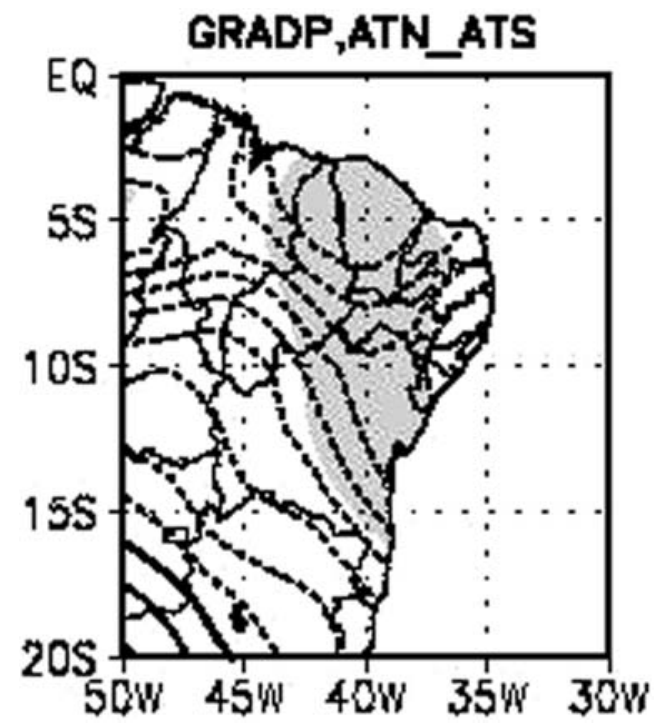

Figura 11 - Médias de anomalias mensais de precipitação em MAM para composição de GRADP/ATN_ATS. A convenção gráfica é igual a da Figura 3.

\section{DISCUSSÕES E CONCLUSÕES}

O presente trabalho re-examina a relação da variabilidade de precipitação na região da América do Sul limitada entre o equador, $20^{\circ} \mathrm{S}, 50^{\circ} \mathrm{W}$ e $30^{\circ} \mathrm{W}$ com a variabilidade de TSM dos oceanos Pacífico e AT na estação que antecede a estação chuvosa do NEB. Análises para os eventos ENOS isoladamente
(El Niño/s_GRAD e La Niña/s_GRAD) mostram uma tendência de aumento (diminuição) de precipitação na parte norte da região de estudo durante eventos de La Niña (El Niño), como sugerido por trabalhos anteriores. Na ausência de ATSMs significativas no AT, durante a fase inicial e de desenvolvimento do ENOS, as relações da precipitação do NEB e ENOS seguem o caminho esperado, com as anomalias na precipitação em DJF possivelmente associadas aos padrões de teleconexões atmosféricos, que se caracterizam pelo rearranjamento da célula de Walker, e as anomalias de MAM associadas à influência dos padrões de teleconexões extratropicais como discutido por Nobre e Shukla (1996).

Por outro lado, os resultados sugerem que condições de El Niño e GRAD no AT ocorrendo simultaneamente, atuam para aumentar (GRADP) ou diminuir (GRADN) as magnitudes das anomalias de precipitação na parte norte da região em MAM em relação àquelas obtidas considerando somente os efeitos de eventos quentes do Pacífico. Condições de La Niña e GRAD no AT ocorrendo simultaneamente, podem apresentar configurações inversas das obtidas considerando somente os efeitos de eventos La Niña, dependendo da condição do AT. Estes resultados são consistentes com estudos anteriores que mostraram através de modelagem (Pezzi e Cavalcanti, 2001), estudo de casos (Souza et al., 2004) e composições (Ambrizi et al., 2004) que em certas situações o efeito da variabilidade do AT pode sobrepujar o efeito da variabilidade no Pacífico Leste associada ao ENOS, e em outros não. No presente trabalho apresenta-se um detalhamento maior quanto ao sinal do GRAD.

No caso de La Niña/c_GRADP (La Niña/c_GRADN), a região norte do NEB apresenta anomalias negativas (positivas) de precipitação e na região sul do NEB as anomalias são enfraquecidas na estação chuvosa. Neste caso a variabilidade de TSM no AT favorece o posicionamento da ZCIT do AT ao norte (ao sul) de sua posição normal, que por sua vez é mecanismo dinâmico que explica a distribuição de anomalias de precipitação no NEB.

Um aspecto importante nessas análises é o papel do ATS. Quando as ATSMs do ATS apresentam mesmo sinal que as do Pacífico as anomalias de precipitação do norte do NEB são enfraquecidas (como no caso de El Niño/c_GRADN) ou mudam de sinal (La Niña/c_GRADP), ou seja, as ATSMs no ATS agem contrariamente à influência dos padrões de teleconexões associados aos eventos ENOS no AT e NEB. No caso de El Niño/c_GRADN (La Niña/c_GRADP), a presença de ATSMs positivas (negativas) no ATS favorecem o posicionamento da ZCIT do AT ao sul (norte) do equador, que é conflitante com seu posicionamento esperado durante um El Niño (La Niña). Isso contribui para o enfraquecimento das anomalias de precipitação no NEB. 
Nos casos em que as ATSMs do ATS são fracas ou com magnitudes equivalentes daquelas do ATN, porém com o sinal oposto das ATSMs do Pacífico, as ATSMs do ATN e as anomalias da circulação atmosférica relacionadas ao ENOS têm efeitos na precipitação do NEB que se somam, e assim fortalecem os padrões anômalos de precipitação. Nestes casos, a ZCIT do AT apresenta um posicionamento latitudinal esperado em um evento El Niño (ou La Niña) de forma que os efeitos remoto do ENOS e regionalmente da posição da ZCIT se somam.

Consistente com Giannini et al. (2004), os resultados deste trabalho mostram a importância do ATS na variabilidade de precipitação do NEB. O desenvolvimento e a persistência das ATSMs no ATS durante a fase madura do ENOS podem ser cruciais no desenvolvimento das teleconexões associados ao ENOS

Independente do ENOS, a influência do AT na variabilidade de precipitação é mais marcante para a variabilidade de TSM do ATS quando comparada com a do ATN. Esse resultado é consistente com os estudos de Moura e Shukla (1981) e Kane (1992). Para os casos quando as ATSMs do ATN e ATS apresentam valores balanceados, porém de sinais contrários, são notadas anomalias de precipitação de maiores magnitudes em relação aos anomalias associadas aos gradientes definidos por ATSMs intensas no ATN e fracas no ATS, ou intensas no ATS e fracas no ATN. No entanto, a probabilidade de ocorrência desses eventos de dipolo (considerando somente os eventos ocorrendo isoladamente no AT) é de $12 \%$, aproximadamente.

Um ponto relevante apresentado nessas análises é a importância do ATS na variabilidade de precipitação do NEB. Explicar como as ATSMs surgem no ATS foge do escopo do presente trabalho. No entanto, como essas anomalias persistem por alguns meses, o seu monitoramento é essencial para propósitos de previsões climáticas sazonais.

\section{AGRADECIMENTOS}

As autoras agradecem as sugestões dos dois revisores anônimos. A primeira autora agradece o Conselho Nacional de Desenvolvimento Científico e Tecnológico (CNPq), através do processo PD-150.151/04-7, pelo auxílio financeiro recebido. A segunda autora foi parcialmente financiada pelo CNPq.

\section{REFERÊNCIAS BIBLIOGRÁFICAS}

AMBRIZZI, T.; SOUZA, E.B.; PULWARTY, R.S. The Hadley and walker regional circulations and associated ENSO impacts on South American seasonal rainfall. In: THE HADLEY CIRCULATION: PRESENT, PAST AND FUTURE, Diaz H.F. and Bradley R.S. (Eds.). Kluwer Publishers, Chapter 7, p. 203-235, 2004.
ANDREOLI, R.V.; KAYANO, M.T. Multi-scale variability of sea surface temperature in the tropical Atlantic. J. Geophys. Res., v.109, C05009, doi: 10.1029/2003JC002220, 2004.

ANDREOLI, R.V.; KAYANO, M.T.; GUEDES, R.L.; OYAMA, M.D.; ALVES, M.A.S. A influência da temperatura da superfície do mar dos oceanos Pacífico e Atlântico na variabilidade de precipitação em Fortaleza.

Rev. Bras. Meteor., v.19, n.3, p.337-344, 2004.

CHANG, P.; JI, L.; LI H. A decadal climate variation in the tropical Atlantic ocean from thermodynamic air-sea interactions. Nature, v.385, n.6, p.516-518, 1997.

ENFIELD, D.B.; MAYER D.A. Tropical Atlantic SST variability and its relation to El Niño-Southern Oscillation. J. Geophys. Res., v.102, n.C1, p.929-945, 1997.

ENFIELD, D.B.; MESTAS-NUÑEZ, A.M.; MAYER, D.A.; CID-SERRANO, L. How ubiquitous is the dipole relationship in tropical Atlantic sea surface temperature? J. Geophys. Res., v.104, n.C4, p.7841-7848, 1999.

GIANNINI, A.; SARAVANAN, R.; CHANG, P. The preconditioning role of Tropical Atlantic Variability in the development of the ENSO teleconnection: implications for the prediction of Nordeste rainfall. Clim. Dyn., v.22, p. 839-855, doi: 10.1007/s00382004-0420-2, 2004.

HASTENRATH, S. Prediction of Northeast Brazil rainfall anomalies. J. Climate, v.3, n.8, p.893-904, 1990.

HASTENRATH, S.; GREISCHAR, L. Further work on the prediction of Northeast Brazil rainfall anomalies. J. Climate, v.6, n.4, p.743-758, 1993.

HASTENRATH, S.; HELLER, L. Dynamics of climatic hazards in northeast Brazil. Quart. J. Roy. Meteor. Soc., v.103, p.77-92, 1977.

HOREL, J.D.; WALLACE, J.M. Planetary scale atmospheric phenomena associated with the Southern Oscillation. Mon. Wea. Rev., v.109, p. 813-829, 1981.

HOUGHTON, R.W.; TOURRE Y.M. Characteristics of low-frequency sea surface temperature fluctuations in the tropical Atlantic. J. Climate., v.5, n.7, p.765-771, 1992. 
HULME, M. A. 1951-80 global land precipitation climatology for the evaluation of General Circulation Models. Clim. Dyn., v.7, p.57-72, 1992.

HULME, M.A. Validation of large-scale precipitation fields in General Circulation Models. In Global Precipitations and Climate Change, eds. Desbois, M., and F. Desalmand, NATO ASI series, Springer-Verlag, Berlin, 1994. 466 p.

HULME, M.A.; OSBORN, T.J.; JOHNS, T.C. Precipitation sensitivity to global warming: comparison of observations with HadCM2 simulations. Geophys. Res. Lett., v.25, p.3379-3382, 1998.

KANE, R.P. El Niño and La Niña events and rainfall in NE and South Brazil. Rev. Bras. Geofísica, v.10, p.49-59, 1992.

KANE, R.P. Prediction of droughts in north-east Brazil: role of ENSO and use of periodicities. Int. J. Climatol., v.17, p.655-665, 1997.

KAYANO, M.T.; ANDREOLI R.V. Decadal variability of northern northeast Brazil rainfall and its relation to tropical sea surface temperature and global sea level pressure anomalies. J. Geophys. Res., v.109, n.C11011, doi: 10.1029/2004JC002429, 2004.

KAYANO, M.T.; RAO, V.B.; MOURA, A.D. Tropical circulations and the associated rainfall anomalies during two contrasting years. J. Climatol.,v.8, p.477-488, 1988.

KOUSKY, V.E.; KAYANO, M.T.; CAVALCANTI, I.F.A. A review of the Southern Oscillation: oceanic-atmospheric circulation changes and related rainfall anomalies. Tellus, v.36A, p.490-504, 1984.

MARKHAM, C.G.; MCLAIN, D.R. Sea surface temperature related to rain in Ceará, northeastern Brazil. Nature, v.265, p.320-323, 1977.

MO, K.C.; HAKKINEN, S. Interannual variability in the tropical Atlantic and linkages to the Pacific. J. Climate, v.14, n.12, p.2740-2762, 2001.

MOURA, A.D.; SHUKLA J. On the dynamics of droughts in Northeast Brazil: observations, theory, and numerical experiments with a general circulation model. J. Atmos. Sci., v.38, n.12, p.2653-2675, 1981.
NOBRE, P.; SHUKLA J. Variations of sea surface temperature, wind stress, and rainfall over the tropical Atlantic and South America. J. Climate, v.9, n.19, p.2464-2479, 1996.

PEZZI, L.P.; CAVALCANTI, I.F.A. The relative importance of ENSO and tropical Atlantic sea surface temperature anomalies for seasonal precipitation over South America: a numerical study. Clim Dyn., v.17, p.205-212, 2001.

RASMUSSON, E.M.; CARPENTER, T.H. Variations in the tropical sea temperature and surface wind fields associated with the Southern Oscillation/El Niño. Mon. Wea. Rev., v.10, n.5, p.354-384, 1982.

ROPELEWSKI, C.F.; HALPERT, M.S. Global and regional scale precipitation patterns associated with the El Niño/ Southern Oscillation. Mon. Weather Rev., v.115, p.16061626, 1987.

ROPELEWSKI, C.F.; HALPERT, M.S. Precipitation patterns associated with the high index phase of the Southern Oscillation. J. Climate, v.2, p.268-284, 1989.

SERVAIN, J. Simple climatic indices for the tropical Atlantic Ocean and some applications. J. Geophys. Res., v.96, n.C8, p.15,137-15,146, 1991.

SMITH, T. M.; REYNOLDS, R. W. Extended reconstruction of global sea surface temperatures based on COADS data (1854-1997). J. Climate, v.16, p. 1495-1510, 2003.

SARAVANAN, R.; CHANG, P. Interaction between Tropical Atlantic variability and El Niño-Southern Oscillation. J. Climate, v.13, n.13, p. 2177-2194, 2000.

SOUZA, E.B.; AMBRIZZI, T.; COELHO, C.A.S. Two episodes with reversed impacts on the regional precipitation of the northeastern South America. Meteorologica, v.29, n. 1-2, p. 5-16, 2004.

UVO, C.B.; REPELLI, C.A.; ZEBIAK, S.E.; KUSHNIR, Y. The relationships between Tropical Pacific and Atlantic SST and Northeast Brazil monthly precipitation. J. Climate, v.11, p.551-562, 1998.

WALKER, GT. Ceará (Brazil) famines and the general air movement. Beitrage zur Physik der freien Atmosphare, v.14, p.88-93, 1928. 